\title{
EVALUACIÓN DE HYMENOPTERA Y COLEOPTERA (INSECTA) EN DIFERENTES TIPOS DE VEGETACIÓN DE LA ZONA RESERVADA ALLPAHUAYO - MISHANA, LORETO, PERÚ*
}

\author{
Alicia De La Cruz ${ }^{1}$, Paola Aibar ${ }^{2}$, Lilia Campos ${ }^{3}$, Roberto Polo ${ }^{4}$, Sandra Ríos ${ }^{3}$, Gorky Valencia ${ }^{5}$ y José \\ Santisteban $^{6}$
}

\begin{abstract}
RESUMEN
Se analizó la composición de familias de los órdenes Hymenoptera y Coleoptera en cinco diferentes tipos de hábitat, basados en criterios geológicos y de vegetación, en la Zona Reservada Allpahuayo - Mishana, en el marco del curso "Uso de inventarios biológicos para revelar patrones de distribución geográfica de especies en la selva baja amazónica", organizado por el proyecto Diversidad Biológica de la Amazonía Peruana, Perú Finlandia (BIODAMAZ), con el apoyo del Instituto de Investigaciones de la Amazonía Peruana (IIAP) y la Universidad Nacional de la Amazonía Peruana (UNAP). Fueron utilizadas trampas colectoras de insectos tipo Malaise, las cuales fueron colocadas en cinco tipos de formaciones vegetales. Los patrones obtenidos de composición de familias no muestran similitud con los patrones de distribución de las formaciones vegetales evaluadas. Se sugiere evaluar la composición de los órdenes mencionados utilizando un nivel taxonómico más específico (especie) que permita una estimación más precisa.
\end{abstract}

Palabras clave: Amazonía peruana, Coleoptera, formaciones geológicas, Hymenoptera, tipos de vegetación, Zona Reservada Allpahuayo - Mishana.

\begin{abstract}
The composition of families in the Hymenoptera and Coleoptera orders was analyzed in the Allpahuayo-Mishana Reserved Zone, in five different types of habitat based on geological and vegetation criteria, as part of the "Use of biological inventories to reveal geographic distribution patterns of lowland Amazonian species" course organized by the Peru-Finland Biological Diversity of the Peruvian Amazon project (BIODAMAZ), with the support of the Peruvian Amazonian Research Institute (IIAP) and the Peruvian Amazonian National University (UNAP). Malaise traps were used to collect the insects, and these were positioned in five types of forest. The patterns obtained on the composition of families do not show similarity with the distribution patterns of the five forest types. The suggestion is made that the species level is used to evaluate the composition of the mentioned orders in order to get more precise information.
\end{abstract}

Key words: Allpahuayo-Mishana Reserved Zone, Coleoptera, geological formations, Hymenoptera, Peruvian Amazon, types of vegetation.

* Referencia bibliográfica del artículo: De La Cruz, A., Aibar, P., Campos, L., Polo, R., Ríos, S., Valencia, G. \& Santisteban, J. 2002. Evaluación de Hymenoptera y Coleoptera (Insecta) en diferentes tipos de vegetación de la Zona Reservada Allpahuayo - Mishana, Loreto, Perú. En: Rodríguez, J.J., Ruokolainen, K. \& Soini, P. (eds.). La diversidad biológica de la Zona Reservada Allpahuayo - Mishana, Loreto, Perú: Relaciones entre distribución, abundancia y hábitats. Folia Amazónica 14 (1-2): NÚMEROS DE PÁGINA.

1 Universidad Nacional Agraria La Molina (UNALM), Lima, Perú. Correo electrónico: biodamaz@iiap.org.pe

2 Universidad Nacional Agraria La Molina (UNALM), Lima, Perú.

3 Universidad Nacional de la Amazonía Peruana, (UNAP), Iquitos, Perú.

4 Universidad Nacional de Trujillo (UNT), Trujillo, Perú.

5 Universidad Nacional San Antonio Abad del Cusco (UNSAAC), Cusco, Perú.

6 Museo de Historia Natural, Universidad Nacional Mayor de San Marcos (UNMSM), Lima, Perú. 


\section{INTRODUCCIÓN}

Los artrópodos, y en especial los insectos, son los organismos vivos con más éxito sobre la superficie de la tierra. Constituyen un componente importante de diversos ecosistemas, ya que desempeñan funciones importantes en los procesos que rigen los ecosistemas (Wilson, 1987; Miller, 1993; Samways, 1993). Los insectos están involucrados en procesos ecológicos tales como reciclado de materia orgánica en descomposición, transmisión de propágulos y polinización de plantas superiores, control de poblaciones de otros organismos (incluyendo otros insectos), y como integrantes de las cadenas tróficas, debido a su gran diversidad y abundancia (Riechert, 1974; Stork, 1988).

Los patrones de distribución geográfica de especies han sido comúnmente relacionados con condiciones ecológicas actuales; sin embargo, muchas veces estas condiciones ecológicas no explican los patrones observados actualmente. Para comprender los patrones de distribución de especies es necesario incluir en los estudios clásicos la historia geológica y la geomorfología del lugar (Tuomisto y Ruokolainen, 1997).

Los insectos poseen características ideales para ser considerados como grupos indicadores de patrones de distribución geográfica, pues son fáciles de muestrear, poseen especies endémicas y son suficientemente sensibles para medir cambios de biodiversidad.

Los órdenes seleccionados para realizar el estudio fueron Coleoptera e Hymenoptera, por su abundancia y facilidad de colecta con el equipo disponible para esta evaluación, además de ser fácilmente reconocidos a nivel de familias.

Nuestro objetivo fue evaluar si los patrones de abundancia y composición de familias de Hymenoptera y Coleoptera reflejan la distribución de las formaciones vegetales de la Zona Reservada Allpahuayo - Mishana. El trabajo se realizó en el marco del curso "Uso de inventarios biológicos para revelar patrones de distribución geográfica de especies en la selva baja amazónica", organizado por el proyecto Diversidad Biológica de la Amazonía Peruana, Perú - Finlandia (BIODAMAZ) ${ }^{7}$, con el apoyo del Instituto de Investigaciones de la Amazonía Peruana (IIAP) y la Universidad Nacional de la Amazonía Peruana (UNAP).

\section{MATERIAL Y MÉTODO}

Este estudio se llevó a cabo en la Zona Reservada Allpahuayo - Mishana (ZRAM), situada a $25 \mathrm{~km}$ de la ciudad de Iquitos, provincia de Maynas, departamento de Loreto, Amazonía peruana. Esta zona reservada cuenta con una superficie de 57 663,43 ha (INRENA et al., 2000).

Durante los días 19 al 21 de noviembre de 2000, evaluamos mediante trampas tipo Malaise siete lugares ubicados en las siguientes formaciones vegetales de la ZRAM: Bosque de Terraza Fluvial de Suelos Pobres, Bosques de Formación Pebas, Bosques de Planicie de Inundación en Formación Pebas, Bosque de Varillal Húmedo y Bosque de Varillal Seco (Figura 1). Utilizamos una trampa tipo Malaise en las tres primeras formaciones y dos en las formaciones tipo varillal. El muestreo se realizó diariamente, efectuando tres repeticiones por lugar; se obtuvo un total de 21 muestras.

La identificación de los individuos de los órdenes Coleoptera e Hymenoptera a nivel de familia se realizó utilizando las siguientes claves: Lawrence (1994) para Coleoptera, y Goulet y Huber (1993) para Hymenoptera; en este último se trabajó solamente en las determinaciones del suborden Aculeata, por restricciones de tiempo.

El material colectado fue colocado en frascos entomológicos con alcohol al 70\%, previamente etiquetados, para ser dejados en el Departamento de Fauna de la UNAP.

7 Proyecto Diversidad Biológica de la Amazonía Peruana (BIODAMAZ) es un convenio entre los gobiernos del Perú y de Finlandia ejecutado conjuntamente por el Instituto de Investigaciones de la Amazonía Peruana (IIAP) y por el consorcio finlandés formado por la empresa de consultorías ambientales, Biota BD Oy, y por la Universidad de Turku. 


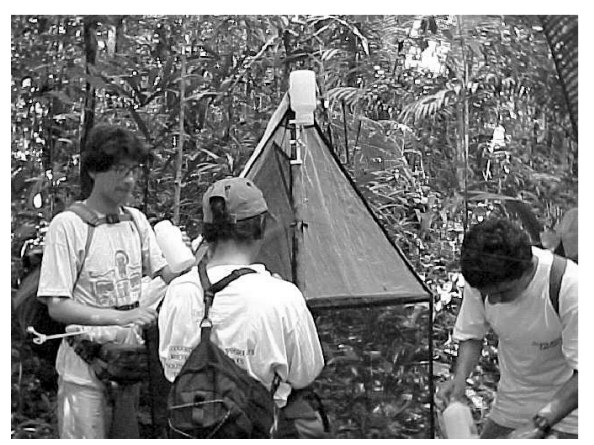

Figura 1. Las trampas Malaise estuvieron ubicadas en los diferentes tipos de hábitats de la Zona Reservada Allpahuayo - Mishana. Este tipo de trampas colecta gran diversidad y cantidad de insectos. Foto: CIIAP-BIODAMAZ/J.J. Rodríguez.

Para evaluar si los patrones de abundancia y composición de familias de los órdenes estudiados reflejan la distribución de las formaciones vegetales de la zona de estudio, se realizó el Análisis de Ordenación de Coordenadas Principales del programa "R-Package", utilizando el índice de correlación de Steinhaus.

\section{RESULTADOS}

Fueron registradas 22 familias del orden Coleoptera, con un total de 425 individuos y 15 familias del orden Hymenoptera, con un total de 809 individuos; a continuación se muestra una lista con las familias identificadas (Tabla 1).

Tabla 1. Familias registradas en el muestreo.

\begin{tabular}{llclc}
\hline & Coleoptera & & Hymenoptera & \\
\hline 1 & Bostrichidae & $\mathrm{N}^{\circ}$ ind. & & $\mathrm{N}^{\circ}$ ind. \\
2 & Carabidae & 1 & Ampulicidae & 2 \\
3 & Cerambycidae & 1 & Apoidea (super familia) & 114 \\
4 & Chrysomelidae & 87 & Bethylidae & 76 \\
5 & Cicindelidae & 2 & Craconidae & 37 \\
6 & Coccinellidae & 2 & Evaniidae & 18 \\
7 & Cucujidae & 2 & Formicidae & 43 \\
8 & Curculionidae & 40 & Ichneumonidae & 391 \\
9 & Elateridae & 6 & Pompilidae & 69 \\
10 & Lampyridae & 8 & Siricidae & 27 \\
11 & Lycidae & 5 & Sphecidae & 2 \\
12 & Melandryidae & 15 & Vespidae & 4 \\
13 & Mordellidae & 6 & Mutilidae & 9 \\
14 & Mycetophagidae & 1 & Tiphiidae & 15 \\
15 & Nitidulidae & 172 & Dryinidae & 1 \\
16 & Pyrochroidae & 10 & & \\
17 & Scaphidiidae & 21 & & \\
18 & Scarabaeidae & 1 & & \\
19 & Scolytidae & 9 & & \\
20 & Silphidae & 1 & & \\
21 & Staphylinidae & 29 & & \\
22 & No determinada & 3 & & \\
& & & & \\
\hline
\end{tabular}


Los gráficos de análisis de ordenación de la composición de familias por fecha, muestran una cierta agrupación, para Varillal Húmedo 1 y Formación Pebas (Figuras 2 y 3). También encontramos que los patrones de similaridad de la composición de familias por hábitats son distintos a los patrones esperados por tipo de formación vegetal (Figuras 4 y 5).

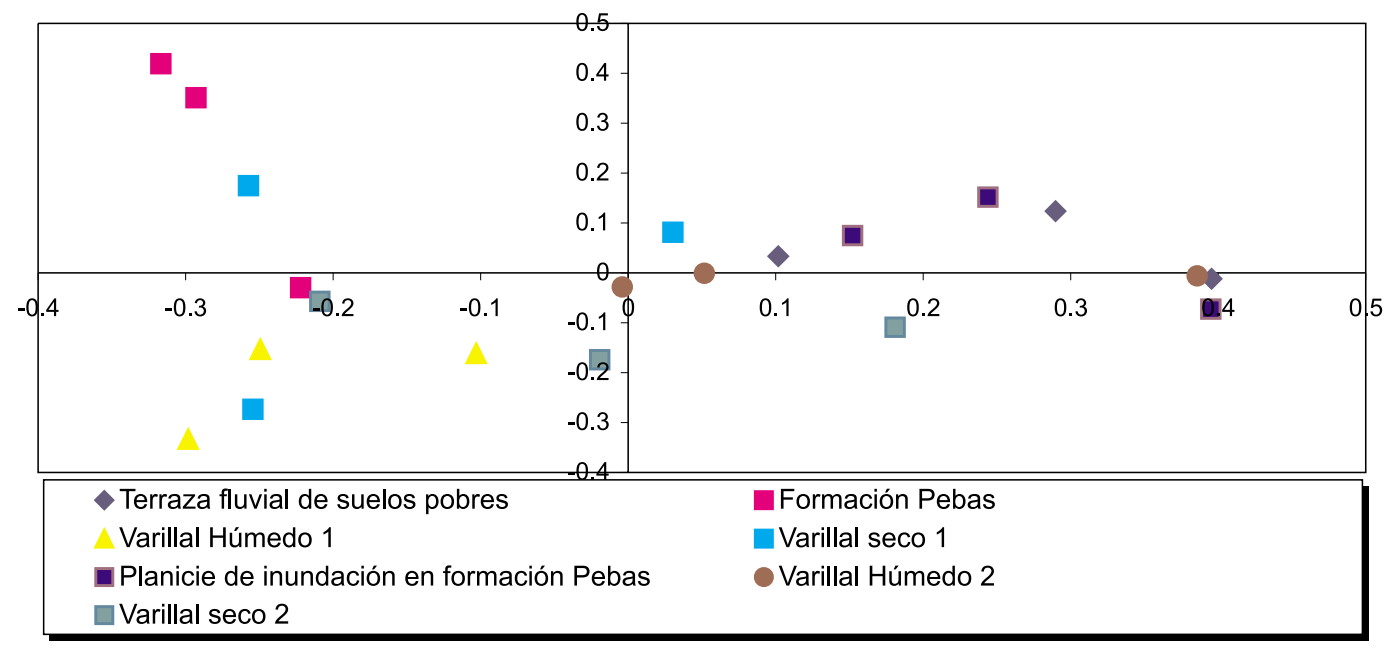

Figura 2. Análisis de ordenación por fecha y lugar de muestreo del orden Coleoptera en la ZRAM, Iquitos, Perú.

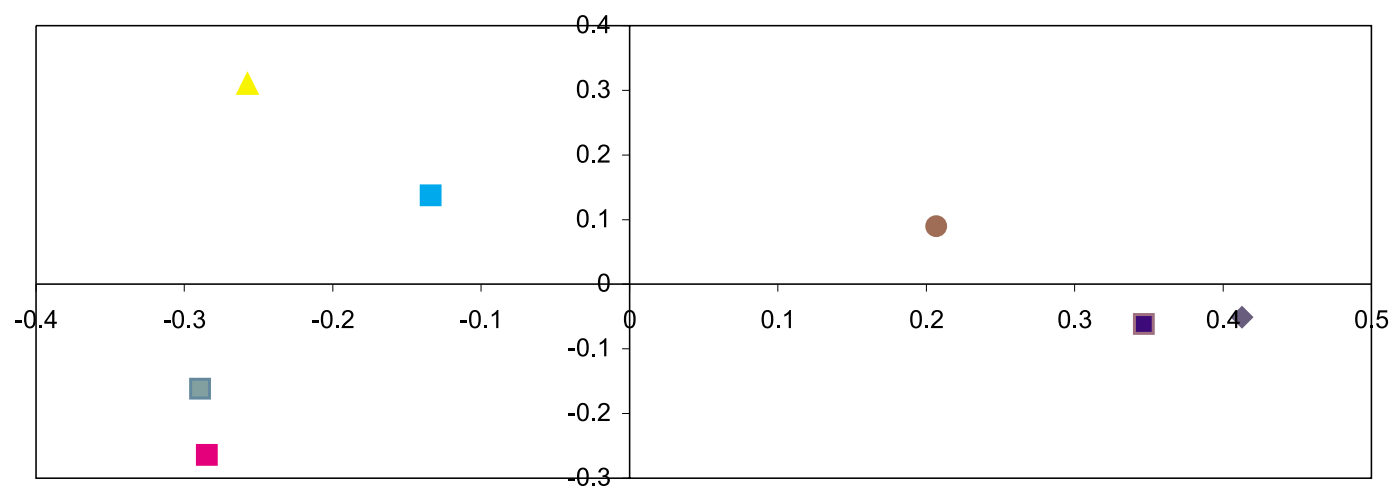

Terraza fluvial de suelos pobres

Varillal Húmedo 1

- Planicie de inundación en formación Pebas

Varillal seco 2
Formación Pebas

- Varillal seco 1

- Varillal Húmedo 2

Figura 3. Análisis de ordenación por fecha y lugar de muestreo del orden Hymenoptera en la ZRAM, Iquitos, Perú. 


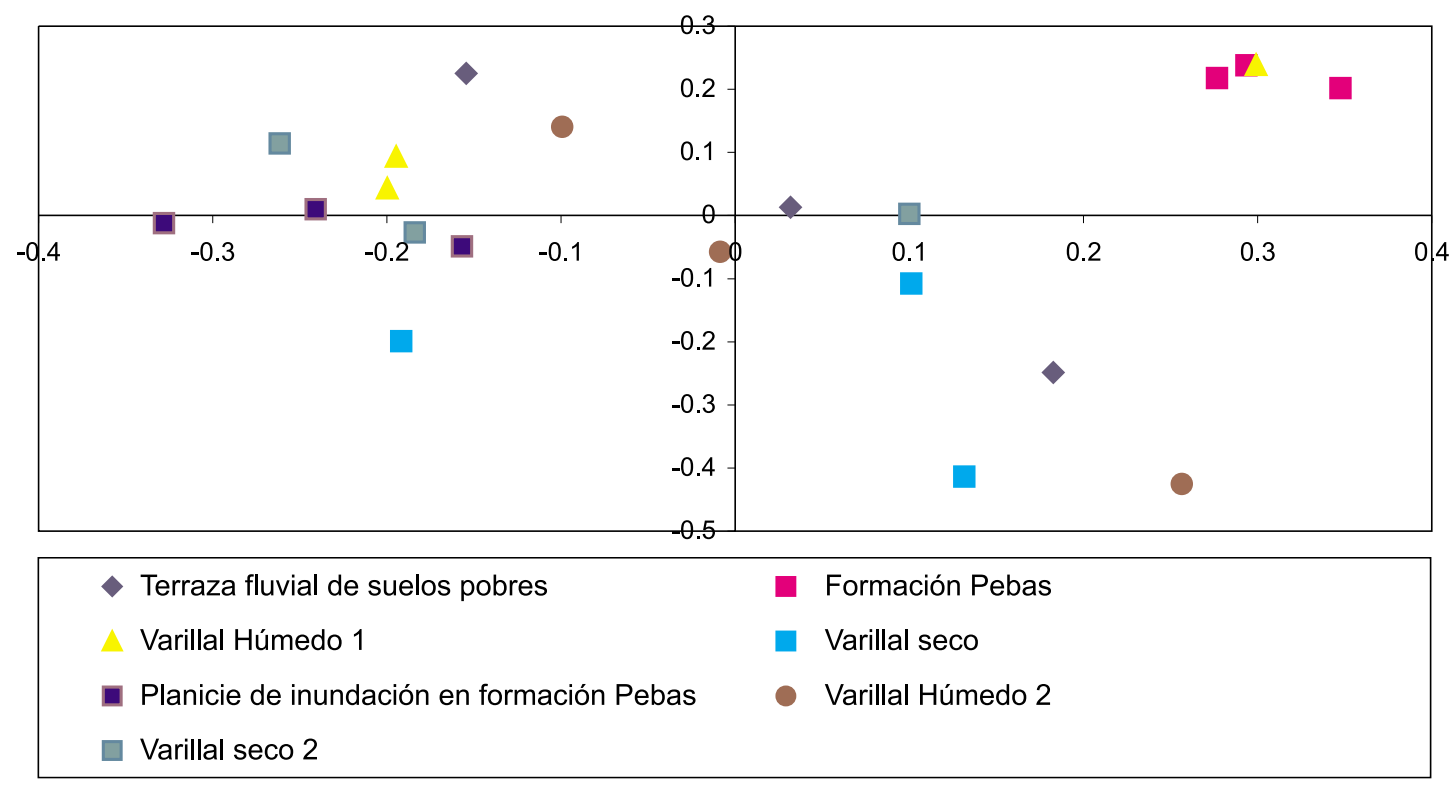

Figura 4. Análisis de ordenación del orden Coleoptera por lugar de muestreo en la ZRAM, Iquitos, Perú.

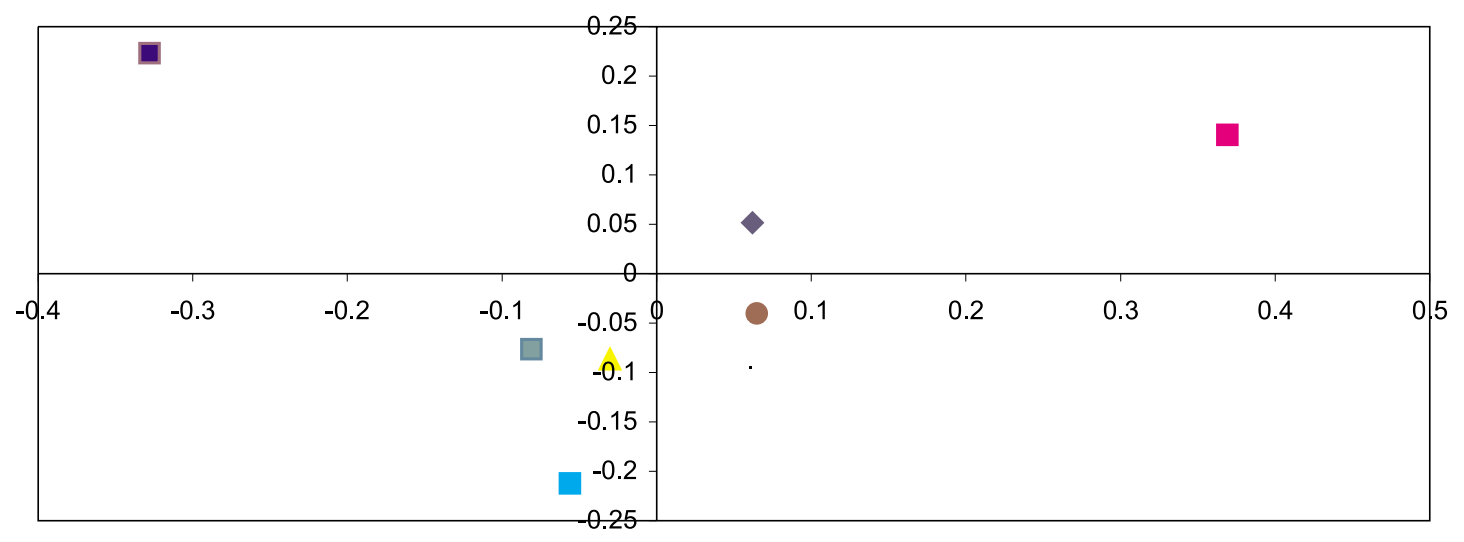

Terraza fluvial de suelos pobres Varillal Húmedo 1

Planicie de inundación en formación Pebas

Varillal seco 2
Formación Pebas

Varillal seco

Varillal Húmedo 2

Figura 5. Análisis de ordenación del orden Hymenoptera por lugar de muestreo en la ZRAM, Iquitos, Perú.

\section{DISCUSIÓN}

La agrupación de los puntos representativos del Varillal Húmedo 1 y Formación Pebas (Figuras 2 y 3), indicaría que el intervalo de tiempo del muestreo fue suficiente. Sin embargo, se ha encontrado que los patrones de similaridad de la composición de familias por hábitat son distintos a los patrones esperados por tipo de formación vegetal, como se puede observar en las Figuras 4 y 5 . En resumen, la estimación encontrada no refleja la 
similaridad de la composición de familias de los órdenes Coleoptera e Hymenoptera con respecto a los hábitats muestreados.

Los resultados presentados podrían deberse a que las familias a las que pertenecen dichos órdenes, colectadas en las trampas Malaise, podrían no ser buenos estimadores para reflejar los patrones de distribución vegetal.

Además, puede ocurrir que la diferencia en composición de familias de una formación vegetal se deba a un reemplazo de ciertas especies por otras con un rol ecológico equivalente.

Por otro lado, pensamos que la composición por especies, cada una con un comportamiento y ecología propia, ofrece un nivel más adecuado de resolución para describir similitudes o distancias entre diferentes formaciones vegetales.

Finalmente, considerando que al nivel de los taxones escogidos, los patrones obtenidos de composición de familias no muestran similitud con los patrones de distribución de las formaciones vegetales evaluadas, recomendamos trabajar los grupos a nivel de especie.

\section{AGRADECIMIENTOS}

Se agradece al equipo de trabajo del proyecto BIODAMAZ, Perú - Finlandia por los esfuerzos en la realización del curso "Uso de inventarios biológicos para revelar patrones de distribución geográfica de especies en la selva baja amazónica"; en especial agradecemos a Kalle Ruokolainen por haber dirigido este estudio y por el constante apoyo brindado durante la redacción del informe. Así mismo, el equipo de trabajo agradece a los organizadores del curso por habernos permitido participar en dicho evento.

\section{BIBLIOGRAFÍA}

GOULET, H.; HUBER, J.T. (eds.). 1993. Hymenoptera of the World: An identification guide to families. Research Branch Agriculture, Canada.

INRENA; IIAP; CTAR-L. 2000. Informe Final de la Comisión Técnica para la Categorización y Delimitación Definitiva de la Zona Reservada Allpahuayo - Mishana. Perú. Sin paginación.

LAWRENCE, J. 1994. Australian Beetles. CSIRO, Australia.

MILLER, J.C. 1993. Insects natural history, multi-species interactions, and biodiversity in ecosystems. Biodiv. Conserv. 2: 233-241.

RIECHERT, S.E. 1974. Thoughts on the ecological significance of spiders. Bioscience 24: 352- 356.

SAMWAYS, M.J. 1993. Insects in biodiversity conservation: some perspectives and directives. Biodiv. Conserv. 2: $258-282$.

STORK, N.E. 1988. Insects diversity: facts, fiction and speculation. Biol. J. Lim. Soc. 35: 321- 353.

TUOMISTO, H.; RUOKOLAINEN, K. 1997. The role of ecological knowledge in explaining biogeography and biodiversity in Amazonia. Biodiv. Conserv. 6: 347-357.

WILSON, E. O. 1987. The little things that run the world: the importance and conservation of invertebrates. Conserv. Biol. 1: 344 - 346. 